\title{
About Control: Kinetics in Molecule- based Photochemical Water Reduction Investigated by Transient IR Spectroscopy
}

\author{
Kerstin T. Oppelt* and Peter Hamm
}

\begin{abstract}
This review aims to promote the role of transient IR spectroscopy to investigate molecular-based photocatalytic water reduction. Examples are discussed in which this method has been successfully applied to elucidate reaction mechanisms. Focus is given to kinetic changes and their consequences when a photochemical water reduction system, which is functional and well understood in solution, is brought onto a metal oxide surface.
\end{abstract}

Keywords: Photocatalytic water reduction - Transient IR spectroscopy

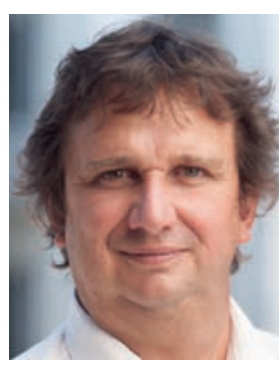

Peter Hamm studied physics at the Technical University of Munich, and carried out his PhD studies under Prof. Dr. W. Zinth at the Ludwig Maximilians University of Munich between 1991 and 1995. He then moved to the University of Pennsylvania, Philadelphia (Prof. R. M. Hochstrasser) for a postdoc. In 1999, he was appointed as an independent group leader at the Max Born Institute, Berlin. In 2001, he moved to the University of Zürich as Associate Professor at the Department of Chemistry, and was promoted to Full Professor in 2007.

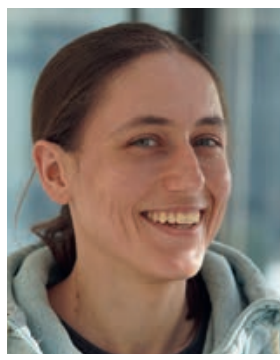

Kerstin T. Oppelt studied Economics of Chemical Engineering at the Technical Faculty of the Johannes Kepler University (JKU) in Linz, Austria. During her PhD in Technical Chemistry, she was a visiting scientist in the Artificial Photosynthesis Group (Dr. Etsuko Fujita) at the Brookhaven National Laboratory. Prof. Serdar Niyazi Sariciftci and Prof. Günther Knör cosupervised her $\mathrm{PhD}$ thesis in Inorganic Photochemistry within the framework of the Solar Fuel Project at the JKU. In 2016, she joined the University Research Priority Program LightChEC at the University of Zürich in the group of Prof. Peter Hamm.

\section{Introduction}

The field of artificial photosynthesis and photochemical water splitting has grown to a substantial size in the last 20 years, as part of an even larger field of research dedicated to renewable fuels, solar energy harvest and storage. The idea of artificial photosynthesis was first introduced by Giacomo Chiamician in 1912. ${ }^{[1]} \mathrm{He}$ already stated that a coal-based society is not sustainable - more than 100 years ago. In that sense, the ideas of artificial photosynthesis and the development of a sustainable energy economy have

${ }^{\star}$ Correspondence: Dr. K. T. Oppelt,

E-mail: kerstin.oppelt@uzh.ch

Department of Chemistry, University of Zurich,

Winterthurerstr. 190,

$\mathrm{CH}-8057$ Zürich, Switzerland been intertwined from the very beginning. ${ }^{[2-5]}$ It is not surprising that this field has kept its relevance also in recent years. ${ }^{6]}$ It is remarkable, however, that no single outstanding solution to this problem exists as of now, rather there are many extremely diverse approaches. The developments in the research of natural photosynthesis, made possible by new spectroscopic and biochemical methods, aided the progress in artificial photosynthesis. ${ }^{[7,8]}$

A large number of review articles on artificial photosynthesis can be found in the literature. In a very recent overview by Licheng Sun and Biaobiao Zhang, molecular catalysis for water splitting is summarized. [9] The application of Re-carbonyl photosensitizers in photochemical water reduction systems has been summarized in ref. [10]. It especially discusses possible advantages to covalently link catalyst and photosensitizer compared to having them separated in solution. The ability to perform and study multielectron processes was found to be crucial as well.[11-13] Parallel to molecular approaches, integrated systems like the artificial leaf, ${ }^{[14-16]}$ dye-sensitized solar cells, ${ }^{[17,18]}$ and spectroscopy of these surfaces ${ }^{[19-22]}$ helped to understand mechanistic fundamentals. Charge transfer processes at the interface in semiconductor-molecular photocatalyst systems have also been reviewed.[23] Modular systems without rare earth metals, using first row transition metal catalysts, is one of the more recent strategic approaches. ${ }^{[24]}$ In this regard, cobalt-based catalysts have gained more interest. ${ }^{[25-30]}$

A systematic investigation of the reaction mechanisms in artificial photosynthesis and photochemical water splitting systems is key for understanding possible design principles for future development of photochemical energy storage systems. There are a variety of available methods, the standards (after basic characterization of the molecules) being UV/VIS absorption and fluorescence quenching measurements followed by irradiation experiments. The latter can provide kinetic and mechanistic information to a certain extent by systematic variation(s) of solvent, $\mathrm{pH}$ value, and relative concentrations of the reagents. Electrochemical and spectroelectrochemical experiments usually supplement the interpretation of spectroscopic data of possible intermediates in the reaction progress, whereas gas chromatography is applied for the assessment of the overall reaction yield of dihydrogen and/or dioxygen.

Spectroscopic monitoring of changes in a continuously irradiated reaction mixture can be dominated by the intermediate(s) that is/are the slowest to react, because they have the chance to accumulate to detectable levels in the process (provided that their 
absorption coefficients allow observation). The very fast (and often more interesting) reactive intermediates can remain hidden in this picture. That is where time-resolved spectroscopy with short pulsed light sources can be applied. Transient IR spectroscopy is technically more demanding than its UV/VIS counterpart, however, it benefits from its chemical specificity. That is, IR spectroscopy observes (changes in the) vibrations of chemical bonds, rather than the multitude of often unresolved electronic transitions stemming from the molecular orbital structure observed by UV/ VIS spectroscopy.

Water splitting is typically divided into its two half reactions, hydrogen evolution or water reduction vs oxygen evolution or water oxidation. Each of these processes can be categorized into four main steps: charge separation, charge transfer/transport, charge accumulation, and eventually hydrogen or oxygen evolution. The water reduction half reaction is easier, since only two charges need to be accumulated. While water reduction systems in homogenous solution have been demonstrated with reasonable yields and turnover-numbers (TONs), ${ }^{[31-34]}$ it seems inevitable that coupling to the oxidative half reaction will require some sort of compartmentation, since too many very reactive redox-partners would coexist otherwise. Compartmentation can be achieved for instance with the help of functionalized surfaces.

The present review concentrates on photocatalytic water reduction, which has been studied much more thoroughly. The individual components, which make up a water reduction system, usually are a photosensitizer (PS), an electron donor (ED) or redox mediator and a water reduction catalyst (WRC). The PS interacts with light. The ED provides the necessary charges. The water reduction catalyst, provided with electrons from the PS and protons from solution, can finally release dihydrogen. In some cases, PS and WRC are combined into a single molecular photocatalyst. ${ }^{[10,35]}$

We will discuss the potential of time-resolved infrared spectroscopy to investigate reaction mechanisms of molecule-based water reduction systems. The main focus will be on a water reduction system, whose reaction mechanism is rather well understood in solution, and we will discuss how the reaction mechanism changes once the system is brought onto a surface. Typically, the reaction cycle in water-splitting systems is controlled kinetically. Reaction rates in homogenous systems are diffusion controlled, and can be tuned by concentration, but that might be limited by the solubility of the reagents.

Assembly of reaction partners on a surface enables much higher local concentrations of the reaction partners and as such much shorter intermolecular distances, and therefore may lead to significantly faster reaction rates. ${ }^{[36]}$ Furthermore, a heterogeneous system does not rely on solubility of the reaction partners in the chosen solvent. However, the price to be paid for assembly of the reaction partners on a surface is that also unwanted processes can speed up significantly, as we will see.

Rhenium carbonyl compounds are particularly suited for observation by time-resolved IR spectroscopy. The redox state of a
Re-carbonyl complex can be directly identified via the carbonyl stretch vibrations, which serve as spectroscopic markers. The CO vibrational modes can also report on the solvent environment surrounding the molecules. ${ }^{[37,38]}$ This review is centered around such model systems, using rhenium carbonyl PS as photoactive compounds, paired with cobalt-based WRC. ${ }^{[39-43]}$ The electron donors are varied according to the experimental parameters of the system investigated. Examples and explanations from other systems will be provided alongside the discussion.

\section{Photocatalytic $\mathrm{H}_{2}$ Evolution Systems}

A scheme of the considered water reduction system is shown in Fig. 1, left. The common feature is the use of a Re-tricarbonyl complex as PS and a cobalt complex as WRC. The PS is excited by light, and then reduced in presence of an sacrificial ED (e.g. triethanolamine or ascorbate). The reduced sensitizer can transfer the electron to a cobalt-based WRC. After subsequent protonation, the reduced cobalt complex then forms dihydrogen via a bimolecular pathway. ${ }^{[42]}$ The catalytic cycle closes by the release of dihydrogen and recovery of the initial cobalt(II) complexes. Many reaction steps in this catalytic cycle can be followed by time-resolved IR spectroscopy. That is, we can compare the decay of the excited state vibrations of the Re complex with or without quencher, observe reductive quenching by the appearance of a new set of frequency-shifted bands and infer charge transfer to the cobalt WRC from their subsequent decay. Once a surface is involved, we have to choose which of the components to immobilize. An example for a completely immobilized system utilizes a molecular-based photocathode, in which the electrode (for example $\mathrm{NiO}$ ) serves as an electron donor. In that case, only the electrolyte and proton source are in the liquid phase. In many partially immobilized systems, the electron donor remains in solution, while PS and WRC are co-immobilized. ${ }^{[9,33,44-46]}$ Immobilization of only the PS or the WRC on a surface has been explored as well.[47,48]

An example for a semi-heterogeneous model system is shown in Fig. 1, right, ${ }^{[49]}$ with $\mathrm{ZrO}_{2}$ as redox-inactive substrate. It is our primary example, where the same reaction steps are expected to occur as in a solution-phase system with very similar molecular components. We however found, disappointingly but not completely unexpected, that irradiation experiments failed to see hydrogen evolution, a point to which we will come back later on in this review. In the following, the three main steps, light absorption, charge separation and charge transfer, are discussed in detail. To that end, we will add the various components of a full water reduction system in a step-by-step manner.

\subsection{Light Absorption and Excited State Lifetime}

Fig. 2a shows three typical transient IR spectra of a Retricarbonyl complex at various delay times. Three negative bands are observed, which correspond to three ground state vibrational modes of the molecule in the range between 1850 and $2100 \mathrm{~cm}^{-1}$ :
Fig. 1. Scheme of the basic processes in a solution-phase system with the various molecular components shown on the left, and the corresponding surfacebased system on the right.

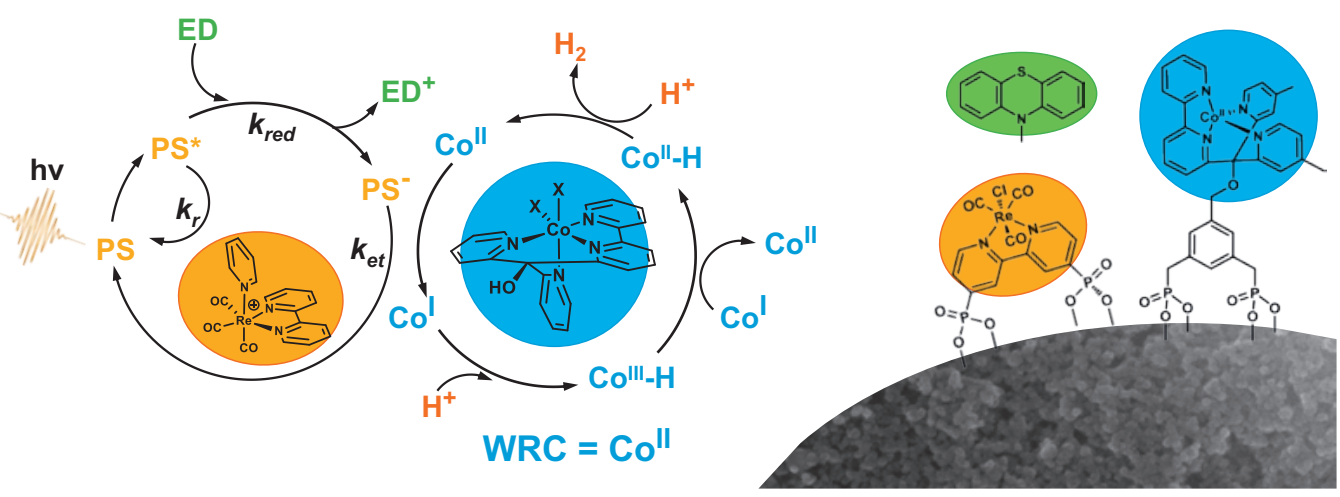


$\mathrm{a}^{\prime}(2)$ (antisymmetric stretching of axial $\mathrm{CO}$ and equatorial $\mathrm{COs}$ ), $\mathrm{a}^{\prime}$ (antisymmetric stretching of the equatorial COs), and $\mathrm{a}^{\prime}(1)$ (symmetric stretching of all COs). ${ }^{[51,52]}$ Light excitation of Rediimine tricarbonyl complexes (most commonly used diimine $=$ 2,2'-bipyridyl or 1,10-phenanthryl) often induces a metal to ligand charge transfer (MLCT) via ultrafast intersystem crossing. This leads to a forbidden transition from the resulting triplet state to the ground state and thus to a relatively long excited state lifetimes in the $10-100$ ns range.[53]
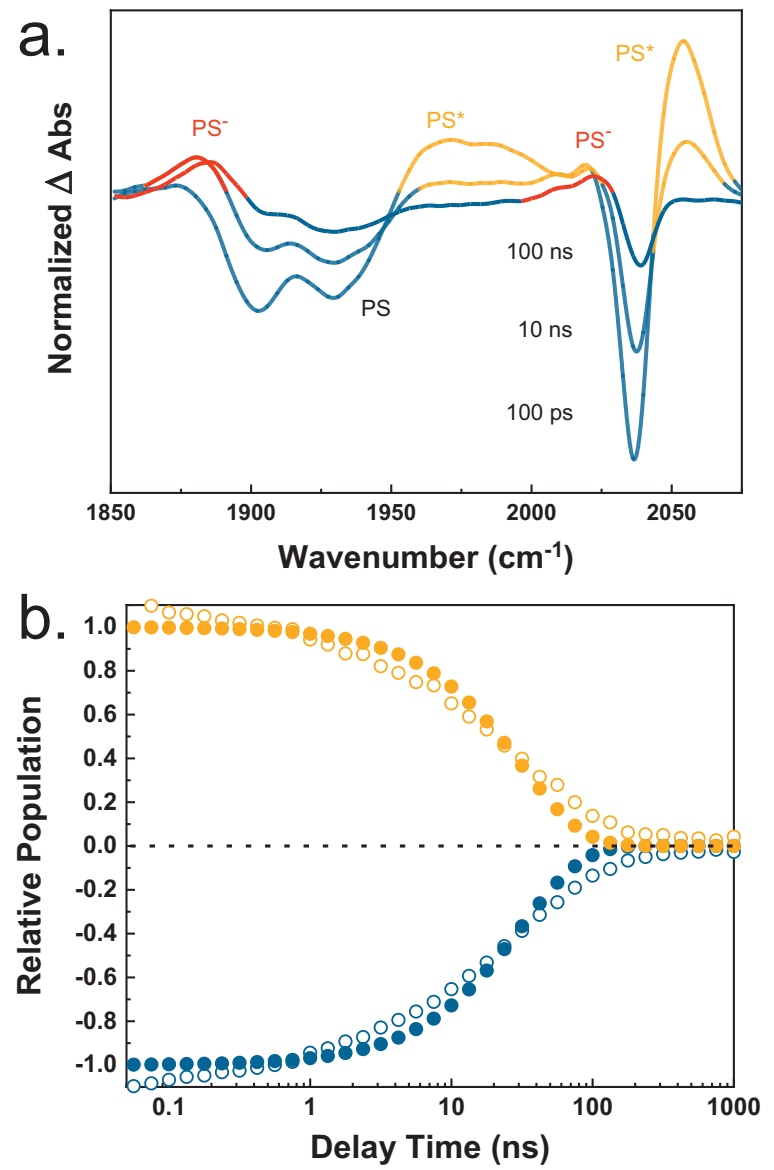

Fig. 2. (a) Typical transient IR measurement of the systems shown in Fig. 1 on $\mathrm{ZrO}_{2}$ surface in the presence of phenothiazine in $\mathrm{EtOH}$ as $\mathrm{ED}$, but without any cobalt WRC. ${ }^{[50]}$ The blue negative part shows the ground state bleach of PS, the yellow positive bands the corresponding bands of PS*, and the positive red signals at later times those of the reduced photosensitizer PS-. (b) Kinetics of exited state absorption signal (yellow) and the ground state bleach (blue) of the systems shown in Fig. 1 on a $\mathrm{ZrO}_{2}$ surface, however without ED and WRC. The full symbols show the results for the solution system, the open circles those of the immobilized system. Adapted with permission from ref. [50]. Copyright (2019) American Chemical Society.

The MLCT process shifts electron density from the Re-center to the aromatic diimine ligand and this influences the strength of back bonding of the CO ligands. All three carbonyl vibrational modes are shifted to higher frequency, resulting in the positive excited state absorption spectrum labelled PS* in Fig. 2a, and indicated in yellow. The opposite frequency shifts are observed upon one electron reduction of the complex (positive bands labelled PS* in Fig. 2a). We see that the CO modes are excellent indicators of the oxidation state of the Re center, without direct involvement of the $\mathrm{CO}$ ligands in the chemistry. Certain other ligands, such as -NCS, change the lowest energy transition into a mixed metal-to-ligand/ligand-to-ligand (MLCT/LLCT) type. Oxidation, reduction and excitation processes of the molecule also change the frequency of corresponding vibrational mode, because the ligand is also participating in back bonding. ${ }^{[39]}$ In very special cases the transition can switch from an MLCT to an intra-ligand type process. Also in these cases, the carbonyl ligands can be used to monitor the change (in this case the increase) of electron density along the ligand and at the Recenter. ${ }^{[54]}$ The description of the spectral response just described is valid both in solution and on a surface.

The intrinsic excited state lifetime is measured in Fig. 2b without the presence of any ED or WRC. In these experiments, one has to keep in mind though, that there is the possibility of triplet-triplet annihilation between two excited PSs on the surface. The laser power, which determines the fraction of excited $\mathrm{PS}$, has to be kept low enough in surface experiments to suppress this additional relaxation channel. The excited state lifetime is strongly affected by the anchoring substituents as well as the solvent. In the case of protic anchoring substituents, also the $\mathrm{pH}$ of the solvent has a strong effect. ${ }^{[50]}$ But these factors affect the excited state lifetime in roughly the same way, regardless whether the system is in solution or surface-immobilized (as long as the surface is redox-inactive, such as $\mathrm{ZrO}_{2}$ ). The biggest difference is the more stretched relaxation kinetics on the surface (Fig. $2 \mathrm{~b}$, open vs closed circles), reflecting the heterogeneity in local environments on a rough surface. Stretched exponential kinetics have also been seen for ruthenium trisbipyridine dyes on large bandgap semiconductor surfaces. ${ }^{[19]}$

This leads us to the next section, the discussion of excited state quenching reactions. For this second step in the reaction cycle, the excited state lifetime defines a time-window, during which another molecule can interact with the excited state and make use of its energy. A long excited state lifetime is therefore desired; provided in the Re-complexes by the forbidden MLCT transition.

\subsection{Quenching of the Exited State}

Bimolecular quenching processes, i.e. excited state deactivation by another molecule, follow various mechanisms, ${ }^{[55]}$ most commonly excitation energy transfer or electron transfer to or from a quencher molecule. The crucial point here is that photochemical hydrogen evolution relies on the fact that the photosensitizer, in our case the Re-carbonyl complex, is quenched either oxidatively or reductively, depending on the relative electrochemical potentials of the reaction partners. All other quenching processes, in particular excited state deactivation via Dexter energy transfer (see below), will not lead to the formation of hydrogen or any other product. The ratio of charge transfer to the PS vs. all other (non-productive) quenching mechanisms, i.e. the quantum yield of $\mathrm{PS}^{-}$formation in our case, is thus a key parameter for the efficiency of a water reduction system.

Reduction of the PS can be studied extremely well by timeresolved IR spectroscopy, observing the bands colored in red in Fig. 2a. The black data in Fig. 3 shows the kinetics of that signal for the solution-phase system. Almost $40 \%$ of the initially photoexcited PS are reductively quenched by the ED within the first few nanoseconds. The population of PS $^{-}$subsequently decays in two steps. We attribute the faster step on a 5 ns timescale to geminate recombination, i.e. to back-electron transfer when both reaction partners are still in the same solvent cage and have not fully separated. It reduces the yield of PS- to about $10 \%$. Only those $\mathrm{PS}^{-}$molecules that do separate from the oxidized $\mathrm{ED}^{+}$can subsequently diffuse and transfer an electron to a WRC and thus contribute to hydrogen evolution. The time window available for this is however limited by non-geminate recombination, i.e. the encounter of a $\mathrm{PS}^{-}$molecule with an oxidized $\mathrm{ED}^{+}$, which had previously separated. In Fig. 3, this non-geminate recombination is reflected by the slower decay of PS $^{-}$population (black curve) on a $5 \mu$ s timescale. 

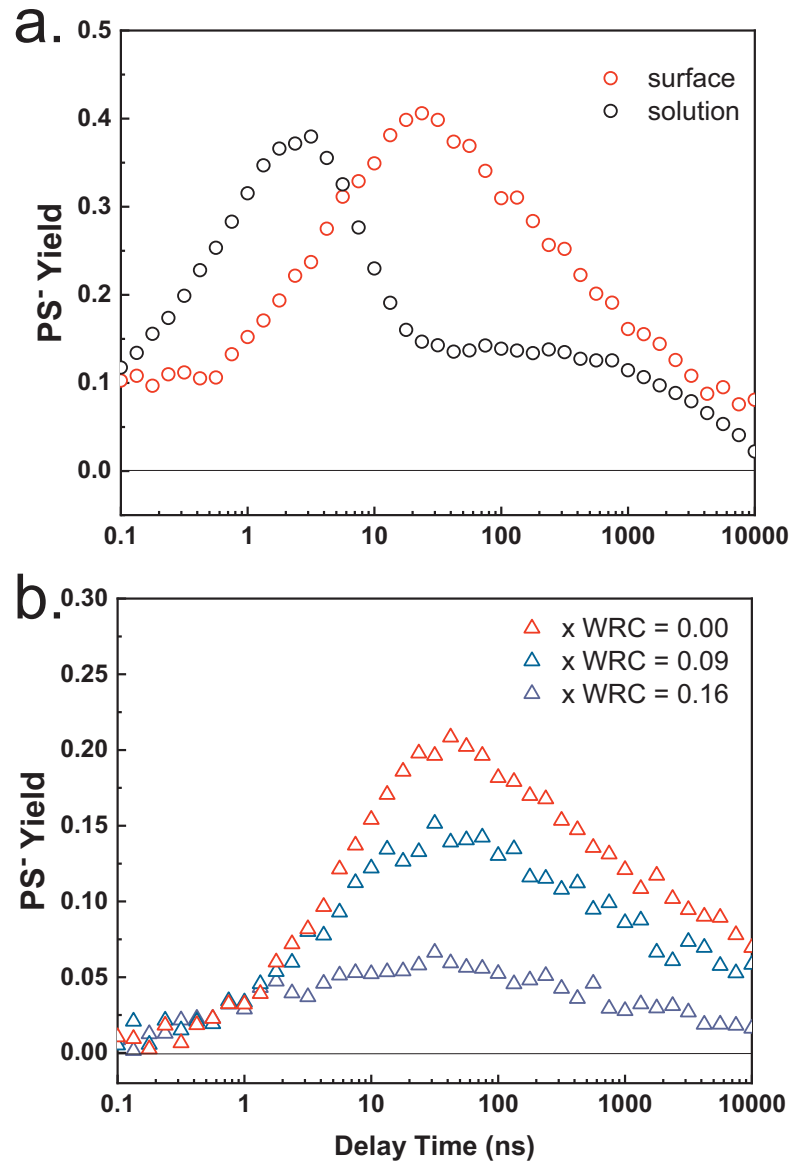

Fig. 3. (a) Kinetic response of reduced PS of the system shown in Fig. 1 , with $100 \mathrm{mM}$ phenothiazine in $\mathrm{EtOH}$ as ED, but without WRC. ${ }^{\text {[50] }}$ The black data show the results for the solution phase system, the red data for the corresponding system on a $\mathrm{ZrO}_{2}$ surface. (b) Kinetic response of reduced PS on a $\mathrm{ZrO}_{2}$ surface with increasing amount of WRC. ${ }^{[49]}$ The red data, in principle, is the same system as in panel (a) without WRC, albeit with slightly different conditions, i.e. a different quencher ( $\mathrm{N}$-methyl-phenothiazine) at lower concentration $(50 \mathrm{mM})$. Panel (a) is adapted with permission from ref. [50]. Copyright (2019) American Chemical Society.

When the PS is bound to a surface, the initial step of reductive quenching remains diffusion-controlled, as the ED is still in solution. We found that the apparent rate of $\mathrm{PS}^{-}$formation for comparable quencher concentrations is slower on the surface by about a factor 6 , as illustrated by the slower rise of the red trace in Fig. 3a. A similar slow-down is observed for geminate recombination, which no longer is clearly distinguishable from the non-geminate decay process.

A slowed-down quenching and geminate recombination rate is actually good news, since it results in a higher PS- concentrations over a larger time-window, as suggested by Fig. 3a. This time-window is available for the next reaction step, electron transfer from $\mathrm{PS}^{-}$to the WRC, discussed in the next section.

\subsection{Electron and Energy Transfer Steps}

Fig. $3 \mathrm{~b}$ shows the amount of surface-bound $\mathrm{PS}^{-}$when coadsorbing WRC. Besides a slower PS- formation due to the lower quencher concentration used in this set of experiments, we observe that the overall PS $^{-}$yield becomes smaller as more WRC is present on the surface. At first sight, this might indicate efficient electron transfer from the reduced $\mathrm{PS}^{-}$to the WRC, which would constitute the desired speed-up of the reaction cycle on the surface. Unfortunately, however, we could not detect any corresponding reduced WRC. ${ }^{[49]}$
To this end, we utilized transient UV/VIS spectroscopy, which reveals a pronounced marker mode of the reduced cobalt WRC at around $620 \mathrm{~nm}$ for the solution phase system. Fig. 4a shows how this band initially rises due to electron transfer from $\mathrm{PS}^{-}$to the WRC, and subsequently decays again due to followup reactions. ${ }^{[42]}$ Fig. $4 \mathrm{~b}$ shows the corresponding experiment on the surface, completely lacking any $\mathrm{Co}^{\mathrm{I}}$ signal. ${ }^{[49]}$ Additionally, as already mentioned above, this system did not produce any hydrogen in irradiation experiments.

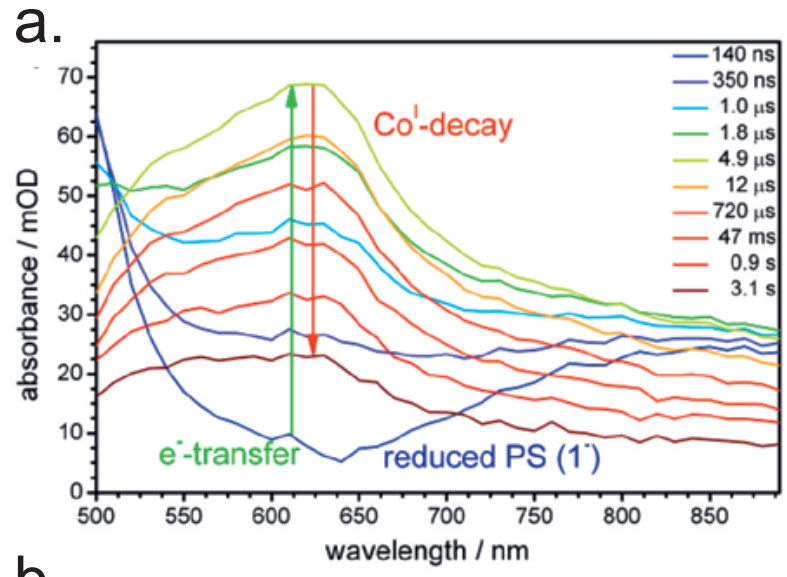

b.

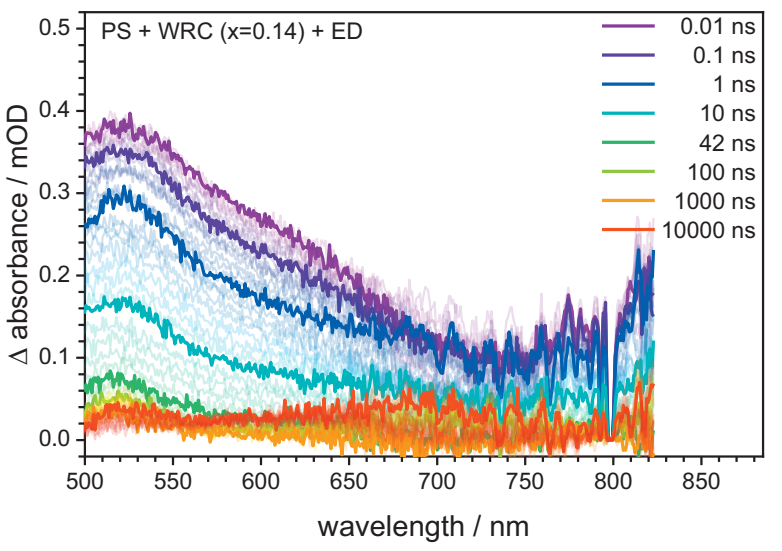

Fig. 4. (a) Transient UV/VIS spectra of PS and WRC in solution. (b) Transient UV/VIS spectra of PS and WRC on $\mathrm{ZrO}_{2}$.[49] Reprinted and adapted with permission from refs. [42] and [49], respectively. Copyright (2020) American Chemical Society.

In order to understand this result, we have to go back and observe the triplet excited state of PS as a function of the amount of WRC on the surface. Fig. 5 shows that the excited state lifetime of PS on the surface decreases dramatically in the presence of WRC, even without any ED. For the extreme case of a WRC/ PS ratio $=1: 1$ (Fig. 5, yellow), the average lifetime decreases by almost one order of magnitude. The suggested non-productive quenching mechanism of PS* by the WRC is a Dexter exchange energy transfer, or a fast electron transfer from PS* to the WRC with almost instant recombination (we cannot distinguish these two processes). In any case, both pathways are short range $(<1 \mathrm{~nm})$ and have an exponential distance dependence, in essence the same as that for the desired charge transfer from PS- to WRC. These non-productive quenching pathways are much faster than of the reaction of PS* with ED necessary to form PS $^{-}$. Since both PS and WRC are distributed on an inhomogeneous surface, there is a wide distribution of intermolecular distances. ${ }^{[49]}$ Hence, for lower WRC/PS ratios, some of the PS* can indeed be reduced by an ED, but they are then too far away from a WRC to reduce it. 


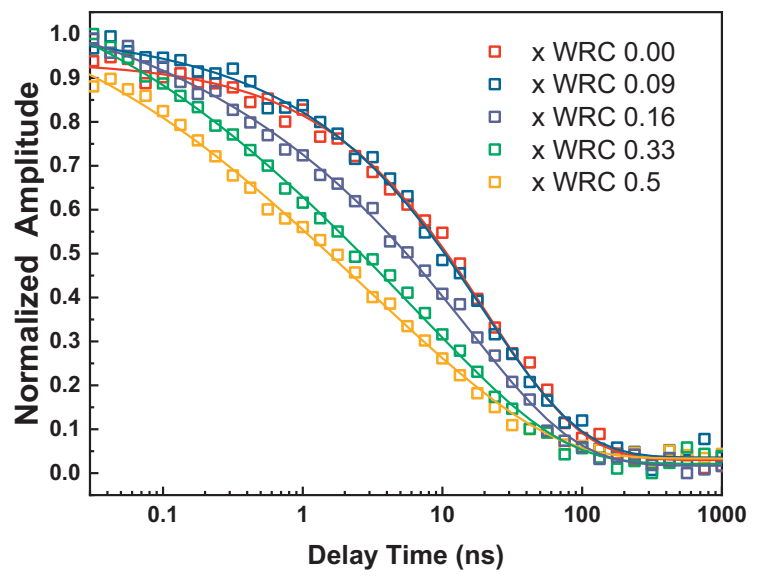

Fig. 5. Excited state decay kinetics of PS* with increasing molar fraction of co-adsorbed WRC. Reprinted with permission from ref. [49]. Copyright (2020) American Chemical Society.

Non-productive quenching of the PS excited state by Dexter energy transfer has also been observed in solution, ${ }^{[39,40]}$ however, the relative concentrations are dramatically different in this case, and that particular loss channel is much less of a problem. To see why, Fig. 6 shows a schematic representation of the distribution of PS (yellow), WRC (blue) and ED (green) for both scenarios. In the solution phase (Fig. 6a), there is a large excess of the ED in comparison to the PS, and the concentration of WRC is typically even lower. This implies that the likelihood of an excited PS being quenched by a WRC is orders of magnitude smaller than by an ED. Once an excited PS is reductively quenched by an ED, excitation energy transfer to the WRC is no longer possible, and productive electron transfer remains the only possibility.
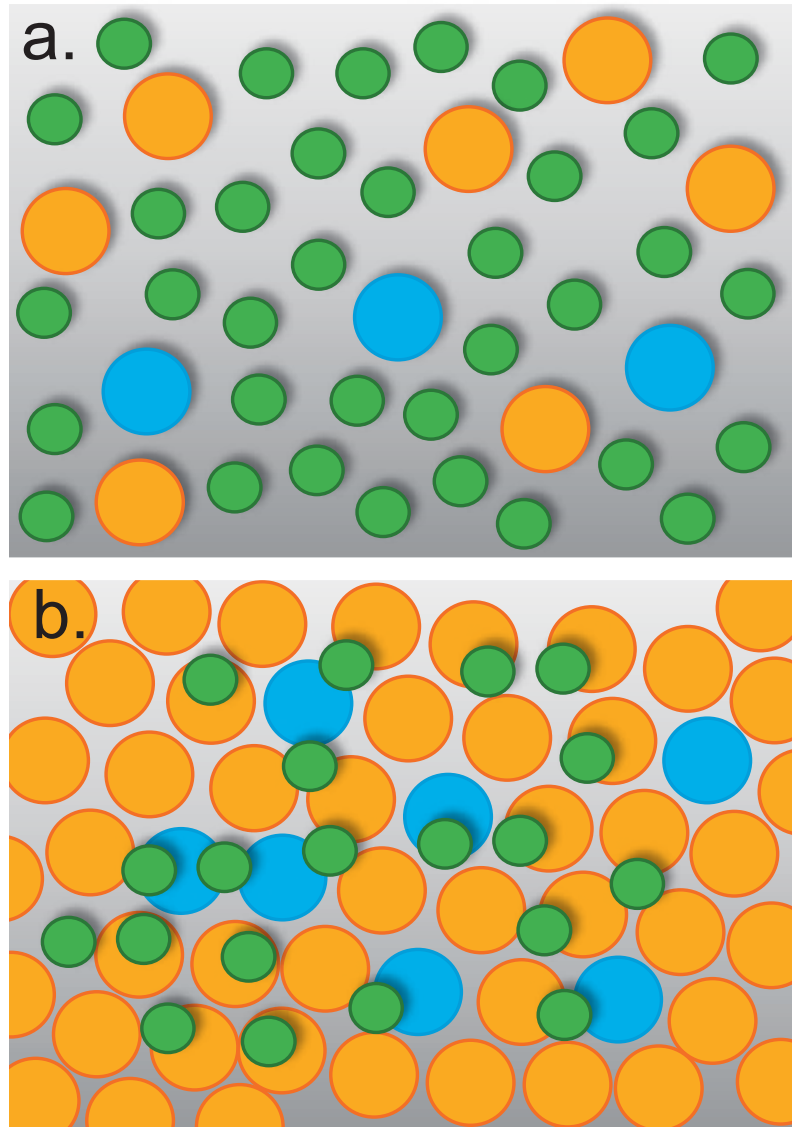

Fig. 6. (a) Distribution of PS (yellow), ED (green) and WRC (blue) in (a) solution and (b) on a densely covered surface.
On the surface (Fig. 6b), this situation is inverted. First, the surface concentrations of PS and WRC are more similar, and electron as well as energy transfer steps between PS and WRC can be very efficient due to the short distances, and are no longer diffusion controlled. On the other hand, while the solution concentration of the ED might still be high, it will be small in comparison to the local concentration of surface immobilized molecules. Quenching by an ED remains diffusion controlled, which is even slower than in homogenous solution phase systems due to spatial restrictions of the surface. Overall, non-productive Dexter energy transfer to the WRC is the dominating effect on the surface, and reductive quenching by the ED cannot compete with this fast process. ${ }^{[49]}$

\section{Outlook}

One way to make sure that a PS is quenched fast is to make the first electron transfer step non-diffusion limited by providing the electron from the substrate. An interesting example in this regard can be found in ref. [36], where a system based on an organic dye photosensitizer in combination with a $\mathrm{Fe}-\mathrm{Fe}$ hydrogenase mimic as WRC on $\mathrm{NiO}$ has been investigated. ${ }^{[36,56]}$

The even bigger, and barely addressed problem is that of charge accumulation. For the formation of dihydrogen, two electrons need to be concentrated on a single catalyst, and even four electrons for dioxygen. In homogeneous solution-phase systems for water reduction, these two electrons can be accumulated by comproportionation in a bimolecular reaction step, see Fig. 1, left. ${ }^{[42]}$ However, this reaction step requires that reduced catalyst molecules are free to diffuse and meet each other. With the WRCs immobilized on a surface, diffusion of the molecules themselves is no longer possible, but a charge might still diffuse via electron transfer hopping between neighboring PS or WRC molecules. We, however, found that this process is not very efficient. ${ }^{[50]}$ Alternatively, charges might diffuse through a conducting substrate, which we consider a very viable route to follow up on. ${ }^{[33,45,57]}$ Finally, ideas have been introduced, where PS and WRC are adsorbed to a silica surface by hydrophobic interactions rather than by covalent bonds. ${ }^{[32]}$ In this way, molecules can still diffuse on the surface, and indeed the system produced dihydrogen.

It is important to note that Nature does not rely on diffusive or bi-molecular processes for charge accumulation. Rather, it works with complex unimolecular constructs, which accumulate reductive equivalents directly. The best example in this regard is the manganese complex of photosystem II. One-and-the-same reaction center subsequently extracts four electrons from this complex, needed to eventually oxidize water. ${ }^{[58]}$ Protected by the protein environment, all redox intermediates in this cycle are stable on a sufficiently long time-scale. Nevertheless, a high current is needed to speed up the cycle, which Nature achieves via large antenna complexes that concentrate the light energy absorbed by many chlorophyll molecules into a single reaction center. On the reductive side, two reductive equivalents are accumulated on quinones in a similar process, whose doubly-reduced state act as electron shuttles. In both cases, these constructs require an extremely high degree of spatial organization, which presently is impossible in artificial systems. Supramolecular constructs have been designed with the goal in mind to steer two reductive equivalents onto a single catalyst, ${ }^{[59-64]}$ but those are very far from any practical applications in artificial photosynthesis at this point.

In conclusion, we deem surfaces necessary to eventually be able to couple the reductive and the oxidative half reactions of water splitting. We have however shown that the direct (or should we say 'naive') transfer of a water reduction system, that has been optimized in homogenous solution, onto a surface will likely not work, since the kinetics of the various reaction steps change by too much. A working water reduction system on a sur- 
face will require different concepts. One possibility is to invoke a semi-conducting substrate as mediator for charge transfer and charge diffusion. Alternatively, we will have to find methods to take more control over the surface arrangement of the reaction partners to better control the kinetics. Both approaches are a playground for a lot of very beautiful science.

\section{Acknowledgements}

We thank our coworkers in this project, in particular Alexander Rodenberg, Margherita Orazietti, Ricardo Fernández-Terán, Roger Alberto and Benjamin Probst. We acknowledge continuous financial support by the Swiss National Science Foundation through Grants CRSII2 136205/1 and CRSII2 160801/1, as well as by the University of Zurich through the URPP LightChEC.

Received: November 23, 2020

[1] G. Ciamician, Science 1912, 36, 385

[2] N. S. Lewis, D. G. Nocera, Proc. Natl. Acad. Sci. U.S.A. 2006, 103, 15729, https://doi.org/10.1073/pnas.0603395103

[3] G. A. Olah, A. Goeppert, G. K. Prakash, 'Beyond Oil and Gas: The Methanol Economy', 2nd Ed., Wiley, 2009, pp. 1-334.

[4] G. Centi, S. Perathoner, ChemSusChem 2010, 3, 195, https://doi.org/10.1002/cssc.200900289

[5] M. Aresta, A. Dibenedetto, A. Angelini, Chem. Rev. 2014, 114, 1709, https://doi.org/10.1021/cr4002758

[6] H. Dau, E. Fujita, L. Sun, ChemSusChem 2017, 10, 4228, https://doi.org/10.1002/cssc.201702106

[7] E. S. Andreiadis, M. Chavarot-Kerlidou, M. Fontecave, V. Artero, Photochem Photobiol. 2011, 87, 946, https://doi.org/10.1111/j.1751-1097.2011.00966.x

[8] A. M. Appel, J. E. Bercaw, A. B. Bocarsly, H. Dobbek, E. L. DuBois, M Dupuis, J. G. Ferry, E. Fujita, R. Hille, P. J. A. Kenis, C. A. Kerfeld, R. H. Morris, C. H. F. Peden, A. R. Portis, S. W. Ragsdale, T. B. Rauchfuss, J. N. H. Reek, L. C. Seefeldt, R. K. Thauer, G. L. Waldrop, Chem. Rev. 2013, 113 , 6621, https://doi.org/10.1021/cr300463y

[9] B. Zhang, L. Sun, Chem. Soc. Rev. 2019, 48, 2216, https://doi.org/10.1039/C8CS00897C

[10] A. Zarkadoulas, E. Koutsouri, C. Kefalidi, C. A. Mitsopoulou, Coord. Chem. Rev. 2015, 304-305, 55, https://doi.org/10.1016/j.ccr.2014.11.006

[11] M. H. V. Huynh, T. J. Meyer, Chem. Rev. 2007, 107, 5004, https://doi.org/10.1021/cr0500030

[12] L. Hammarström, S. Styring, Philos. Trans. R. Soc. Lond., B, Biol. Sci. 2008, 363, 1283, https://doi.org/10.1098/rstb.2007.2225

[13] C. Costentin, J.-M. Savéant, ChemElectroChem 2014, 1, 1226 , https://doi.org/10.1002/celc.201300263

[14] A. J. Esswein , D. G. Nocera, Chem. Rev. 2007, 107, 4022, https://doi.org/10.1021/cr050193e

[15] D. G. Nocera, Acc. Chem. Res. 2012, 45, 767, https://doi.org/10.1021/ar2003013

[16] D. G. Nocera, Acc. Chem. Res. 2017, 50, 616, https://doi.org/10.1021/acs.accounts.6b00615

[17] B. O'Regan, M. Grätzel, Nature 1991, 353, 737.

[18] T. W. Hamann, R. A. Jensen, A. B. F. Martinson, H. Van Ryswyk, J. T. Hupp, Energy Environ. Sci. 2008, 1, 66, https://doi.org/10.1039/B809672D

[19] T. J. Meyer, G. J. Meyer, B. W. Pfennig, J. R. Schoonover, C. J. Timpson, J. F. Wall, C. Kobusch, X. Chen, B. M. Peek, C. G. Wall, W. Ou, B. W. Erickson, C. A. Bignozzi, Inorg. Chem. 1994, 33, 3952, https://doi.org/10.1021/ic00096a020

[20] K. Hu, G. J. Meyer, Langmuir 2015, 31, 11164 https://doi.org/10.1021/acs.langmuir.5b02129

[21] H. W. Bahng, A. Hagfeldt, J. E. Moser, J. Phys. Chem. C 2018, 122, 19348, https://doi.org/10.1021/acs.jpcc.8b04815

[22] T. Lian, M. T. M. Koper, K. Reuter, J. E. Subotnik, J. Chem. Phys. 2019, 150 , 041401, https://doi.org/10.1063/1.5088351

[23] X. Wang, C. Li, J. Photochem. Photobiol. C 2017, 33, 165, https://doi.org/10.1016/j.jphotochemrev.2017.10.003

[24] K. E. Dalle, J. Warnan, J. J. Leung, B. Reuillard, I. S. Karmel, E. Reisner, Chem. Rev. 2019, 119, 2752, https://doi.org/10.1021/acs.chemrev.8b00392

[25] J.-W. Wang, W.-J. Liu, D.-C. Zhong, T.-B. Lu, Coord. Chem. Rev. 2019, 378, 237, https://doi.org/10.1016/j.ccr.2017.12.009

[26] J. L. Dempsey, B. S. Brunschwig, J. R. Winkler, H. B. Gray, Acc. Chem. Res. 2009, 42, 1995, https://doi.org/10.1021/ar900253e

[27] W. T. Eckenhoff, W. R. McNamara, P. Du, R. Eisenberg, Biochim. Biophys. Acta 2013, 1827, 958, https://doi.org/10.1016/j.bbabio.2013.05.003

[28] N. Queyriaux, R. T. Jane, J. Massin, V. Artero, M. Chavarot-Kerlidou, Coord. Chem. Rev. 2015, 304-305, 3, https://doi.org/10.1016/j.ccr.2015.03.014
[29] E. S. Rountree, D. J. Martin, B. D. McCarthy, J. L. Dempsey, ACS Catal. 2016, 6, 3326, https://doi.org/10.1021/acscatal.6b00667

[30] S. Schnidrig, C. Bachmann, P. Müller, N. Weder, B. Spingler, E. Joliat-Wick, M. Mosberger, J. Windisch, R. Alberto, B. Probst, ChemSusChem 2017, 10 , 4570, https://doi.org/10.1002/cssc.201701511

[31] C. Bachmann, B. Probst, M. Guttentag, R. Alberto, Chem. Commun. 2014 50, 6737, https://doi.org/10.1039/C4CC01500B

[32] C. Bachmann, B. Probst, M. Oberholzer, T. Fox, R. Alberto, Chem. Sci. 2016, 7, 436, https://doi.org/10.1039/C5SC02124C

[33] M. A. Gross, A. Reynal, J. R. Durrant, E. Reisner, J. Am. Chem. Soc. 2014 136, 356, https://doi.org/10.1021/ja410592d

[34] W. T. Eckenhoff, Coord. Chem. Rev. 2018, 373, 295, https://doi.org/10.1016/j.ccr.2017.11.002

[35] S. S. Nurttila, R. Becker, J. Hessels, S. Woutersen, J. N. Reek, Chem. Eur. 2018, 24, 16395, https://doi.org/10.1002/chem.201803351

[36] M. G. Gatty, S. Pullen, E. Sheibani, H. Tian, S. Ott, L. Hammarström, Chem. Sci. 2018, 9, 4983, https://doi.org/10.1039/C8SC00990B

[37] D. M. Dattelbaum, T. J. Meyer, J. Phys. Chem. A 2002, 106, 4519, https://doi.org/10.1021/jp014057z

[38] A. El Nahhas, A. Cannizzo, F. van Mourik, A. M. Blanco- Rodríguez, S. Zális, A. Vlcek, M. Chergui, J. Phys. Chem. A 2010, 114, 6361, https://doi.org/10.1021/jp101999m

[39] B. Probst, A. Rodenberg, M. Guttentag, P. Hamm, R. Alberto, Inorg. Chem 2010, 49, 6453, https://doi.org/10.1021/ic100036v

[40] B. Probst, M. Guttentag, A. Rodenberg, P. Hamm, R. Alberto, Inorg. Chem. 2011, 50, 3404, https://doi.org/10.1021/ic102317u

[41] M. Guttentag, A. Rodenberg, R. Kopelent, B. Probst, C. Buchwalder, M. Brandstätter, P. Hamm, R. Alberto, Eur. J. Inorg. Chem. 2012, 59 , https://doi.org/10.1002/ejic.201100883

[42] A. Rodenberg, M. Orazietti, B. Probst, C. Bachmann, R. Alberto, K. K. Baldridge, P. Hamm, Inorg. Chem. 2015, 54, 646, https://doi.org/10.1021/ic502591a

[43] A. Rodenberg, M. Orazietti, M. Mosberger, C. Bachmann, B. Probst, R. Alberto, P. Hamm, ChemPhysChem 2016, 17, 1321, https://doi.org/10.1002/cphc.201501085

[44] H. Tian, ChemSusChem 2015, 8, 3746, https://doi.org/10.1002/cssc.201500983

[45] K. L. Orchard, D. Hojo, K. P. Sokol, M.-J. Chan, N. Asao, T. Adschiri, E. Reisner, Chem. Commun. 2017, 53, 12638 , https://doi.org/10.1039/C7CC05094A

[46] J. Willkomm, N. M. Muresan, E. Reisner, Chem. Sci. 2015, 6, 2727 , https://doi.org/10.1039/C4SC03946G

[47] M. Guttentag, A. Rodenberg, C. Bachmann, A. Senn, P. Hamm, R. Alberto, Dalton Trans. 2013, 42, 334, https://doi.org/10.1039/C2DT31699D

[48] N. Weder, B. Probst, L. Sévery, R. J. Fernández-Terán, J. Beckord, O. Blacque, S. D. Tilley, P. Hamm, J. Osterwalder, R. Alberto, Catal. Sci. Technol. 2020, 10, 2549, https://doi.org/10.1039/D0CY00330A

[49] K. Oppelt, M. Mosberger, J. Ruf, R. Fernández-Terán, B. Probst, R. Alberto, P. Hamm, J. Phys. Chem. C 2020, 124, 12502, https://doi.org/10.1021/acs.jpcc.0c02556

[50] K. Oppelt, R. Fernández-Terán, R. Pfister, P. Hamm, J. Phys. Chem. C 2019, 123, 19952, https://doi.org/10.1021/acs.jpcc.9b04950

[51] J. B. Asbury, Y. Wang, T. Lian, Bull. Chem. Soc. Jpn. 2002, 75, 973, https://doi.org/10.1246/bcsj. 75.973

[52] J. Bredenbeck, J. Helbing, P. Hamm, J. Am. Chem. Soc. 2004, 126, 990 , https://doi.org/10.1021/ja0380190

[53] A. Cannizzo, A. M. Blanco-Rodríguez, A. El Nahhas, J. Sebera, S. Zális, A. Vlcek, M. Chergui, J. Am. Chem. Soc. 2008, 130, 8967, https://doi.org/10.1021/ja710763w

[54] R. Fernández-Terán, L. Sévery, Inorg. Chem. 2020, https://doi.org/10.1021/acs.inorgchem.0c01939.

[55] V. Balzani, P. Ceroni, A. Juris, in 'Photochemistry and Photophysics Concepts, Research, Applications', 1st Ed., WILEY-VCH Verlag \& Co. KGaA, Weinheim, 2014, Chap. 6, pp. 139-168.

[56] M. Mirmohades, A. Adamska-Venkatesh, C. Sommer, E. Reijerse, R. Lomoth, W. Lubitz, L. Hammarström, J. Phys. Chem. Lett. 2016, 7, 3290, https://doi.org/10.1021/acs.jpclett.6b01316

[57] A. M. Brown, L. J. Antila, M. Mirmohades, S. Pullen, S. Ott, L. Hammarström, J. Am. Chem. Soc. 2016, 138, 8060, https://doi.org/10.1021/jacs.6b03889

[58] J. Barber, Chem. Soc. Rev. 2009, 38, 185, https://doi.org/10.1039/B802262N

[59] M. P. O’Neil, M. P. Niemczyk, W. A. Svec, D. Gosztola, G. L. Gaines, M. R. Wasielewski, Science 1992, 257, 63, https://doi.org/10.1126/science.257.5066.63

[60] H. Imahori, M. Hasegawa, S. Taniguchi, M. Aoki, T. Okada, Y. Sakata, Chem. Lett. 1998, 27, 721, https://doi.org/10.1246/cl.1998.721

[61] S. Karlsson, J. Boixel, Y. Pellegrin, E. Blart, H. C. Becker, F Odobel, L. Hammarström, Faraday Discuss. 2012, 155, 233, https://doi.org/10.1039/C1FD00089F

[62] A. G. Bonn, O. Yushchenko, E. Vauthey, O. S. Wenger, Inorg. Chem. 2016, 55, 2894, https://doi.org/10.1021/acs.inorgchem.5b02757 
[63] M. Orazietti, M. Kuss-Petermann, P. Hamm, O. S. Wenger, Angew. Chem. Int. Ed. 2016, 55, 9407, https://doi.org/10.1002/anie.201604030

[64] A. Arrigo, G. La Ganga, F. Nastasi, S. Serroni, A. Santoro, M.-P. Santoni, M. Galletta, S. Campagna, F. Puntoriero, C. R. Chim. 2017, 20, 209, https://doi.org/10.1016/j.crci.2016.02.011
License and Terms

This is an Open Access article under the (C) terms of the Creative Commons Attribution License CC BY 4.0. The material may not be used for commercial purposes.

The license is subject to the CHIMIA terms and conditions: (http:// chimia.ch/component/sppagebuilder/?view=page \&id=12).

The definitive version of this article is the electronic one that can be found at https://doi.org/10.2533/chimia.2021.188 